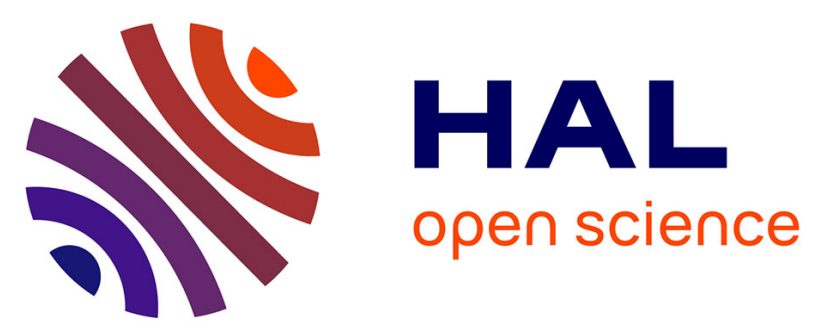

\title{
Cache-Based Side-Channel Attacks Detection through Intel Cache Monitoring Technology and Hardware Performance Counters
}

Mohammad-Mahdi Bazm, Thibaut Sautereau, Marc Lacoste, Mario Südholt, Jean-Marc Menaud

\section{To cite this version:}

Mohammad-Mahdi Bazm, Thibaut Sautereau, Marc Lacoste, Mario Südholt, Jean-Marc Menaud. Cache-Based Side-Channel Attacks Detection through Intel Cache Monitoring Technology and Hardware Performance Counters. FMEC 2018 - Third IEEE International Conference on Fog and Mobile Edge Computing, Apr 2018, Barcelona, Spain. pp.1-6, 10.1109/FMEC.2018.8364038 . hal$01762803 \mathrm{v} 2$

\section{HAL Id: hal-01762803 \\ https://hal.inria.fr/hal-01762803v2}

Submitted on 14 Sep 2018

HAL is a multi-disciplinary open access archive for the deposit and dissemination of scientific research documents, whether they are published or not. The documents may come from teaching and research institutions in France or abroad, or from public or private research centers.
L'archive ouverte pluridisciplinaire HAL, est destinée au dépôt et à la diffusion de documents scientifiques de niveau recherche, publiés ou non, émanant des établissements d'enseignement et de recherche français ou étrangers, des laboratoires publics ou privés. 


\section{Cache-Based Side-Channel Attacks Detection}

\section{through Intel Cache Monitoring Technology}

\section{and Hardware Performance Counters}

Mohammad-Mahdi Bazm*, Thibaut Sautereau*, Marc Lacoste*, Mario Südholt ${ }^{\dagger}$, Jean-Marc Menaud ${ }^{\dagger}$

*Orange Labs, 44 Avenue de la République, 92320 Châtillon, France

Email:mohammadmahdi.bazm@orange.com, thibaut.sautereau@orange.com, marc.lacoste@orange.com

${ }^{\dagger}$ IMT Atlantique, 4 Rue Alfred Kastler, 44307 Nantes, France

Email:mario.sudholteimt-atlantique.fr, jean-marc.menaudeimt-atlantique.fr

\begin{abstract}
Research on cache-based side-channel attacks shows the security impact of these attacks on cloud computing. Therefore, the detection of cache-based sidechannel attacks has received more attention in IaaS cloud infrastructures because of improvements in the attack techniques. However, such detection requires high resolution information, and it is also a challenging task because of the fine-granularity of the attacks. In this paper, we present an approach to detect cross-VM cache-based side-channel attacks through using hardware fine-grained information provided by Intel Cache Monitoring Technology (CMT) and Hardware Performance Counters (HPCs) following the Gaussian anomaly detection method. The approach shows a high detection rate with $2 \%$ performance overhead on the computing platform.
\end{abstract}

Index Terms-side-channel attacks detection, Intel cache monitoring technology, hardware performance counters, cloud security, isolation.

\section{INTRODUCTION}

Cloud computing makes use of virtualization technology for dynamic resource allocation, consolidation and service provisioning. The virtualization layer schedules allocation of hardware resources among virtual instances such as virtual machines (VMs) with different operating systems. However, this layer has shown several security issues related to itself and virtualized instances. Although, the virtualization layer provides isolation, one new challenge is to guarantee strong isolation between virtualized instances running on top of the virtualization layer. OS and hypervisor traditionally implement resource isolation between processes or VMs using software/hardware techniques, such as memory management and access control to key shared physical resources. However, sharing hardware resources has an impact on isolation in virtualized environments. Furthermore, physical resources are often shared between independent users at very low-level through the virtualization layer. Such sharing allows bypassing security mechanisms implemented at virtualization layer, breaking isolation, and threatening the security of the overall virtualized infrastructure. Side-channel attacks 
(SCA) threaten isolation of virtualized environments. These attacks use a leaky channel to obtain secret information such as cryptographic keys. Such channels are either established through techniques or optimization mechanisms e.g., memory deduplication used in the virtualization layer, or micro-architectural vulnerabilities in processors. The threat of side-channel attacks is becoming a security concern in cloud computing [?]. For instance, in IaaS platforms, a CPU cache which is shared between VMs leaks information on cache access patterns of running instances (cross-VM side-channel attacks). The Last-Level Cache (LLC) is one of resources shared among all cores of processor, and leaks cache access patterns of a VM running on another processor core than adversary [2] [3]. To obtain cache access patterns, an adversary does not need to any special privileged access. This is why these attacks gained attention in cloud infrastructures.

Detection of side-channel attacks is hard because of relying on fine-grained information without exploiting special security barriers. These attacks may be detected at OS/hypervisor level through fine-grained information which are often provided by Hardware Performance Counters (HPCs): special platform hardware registers used to store statistics about various events of the processor (e.g., number of cache misses). Existing approaches [4] [5] [6] use only HPCs as the source of raw information in their works to detect cache-based side-channel attacks. However, other hardware technologies such as Intel Cache Monitoring Technology (CMT) providing fine-grained information on the cache behavior of a virtual machine, might be useful to improve the precision of detection approaches. In this work, we present a cross-VM side-channel attacks detection approach. It leverages Intel CMT and HPCs to detect side-channel attacks conducted between two VMs through the LLC. We made the following contributions: (1) we present a prototype to detect side-channel attacks using HPCs and Intel CMT for the first time; (2) we introduce the concept and implementation of the prototype; (3) we evaluate the prototype in terms of security and performance characteristics.

This paper is organized as follows. In Section ??, we give an overview on existing approaches. Then, in Section III, we provide some background on the cache architecture in processors and on cache-based side-channel attacks. In Section III, we give an overview on the design and implementation of the approach. In Section IV we evaluate the implemented prototype. Finally, we conclude with some perspectives in Section $\mathrm{V}$

\section{RELATED WORK}

Almost all the current detection approaches take advantage of Hardware Performance Counters (HPCs) in different ways to detect side-channel attacks. The reason for using HPCs is that they provide information by hardware to detect such fine-grained attacks. As a thresholdbased detection approach; Chiappetta et al. [4] proposed a detection approach leveraging HPCs to detect the attacks. To analyze statistics collected through HPCs, they used machine learning and anomaly detection techniques to detect a side-channel attack between two processes (i.e., spy and victim). As signature-based detection approaches; Zhang et al. [5] presented a real-time detection framework called CloudRadar to detect side-channel attacks between two VMs in the cloud. Their detection approach is based on the correlation between running a cryptographic application in the victim VM and then monitoring the malicious VM. They generate a signature for the sensitive application running in the victim VM, 
by calculating the Fisher Score [7] of different events provided by HPCs. The signature is then used by the detector to identify when the sensitive application is running. Once the application is identified, the detector starts to analyze performance events (e.g., LLC-misses) of the adversary VM to detect any malicious behavior through anomaly detection. Payer et al. [6] also proposed HexPADS to detect inter-process cache-based side-channel attacks. HexPADS generates signatures for cache-based side-channel attacks, using HPCs. It then analyzes running processes in the system to detect which processes have the same signature as the generated signatures.

\section{BACKGROUND}

\section{A. Cache Architecture in Modern Processors}

Caches of CPUs are specialized memory layers located between the CPU and the main memory. They are used to significantly improve the execution efficiency by reducing the speed mismatch between the CPU (operates in $\mathrm{GHz}$ clock cycles) and RAM (requires hundreds of clock cycles to be accessed). In modern processors, we can typically distinguish different levels of cache: L1, L2 and L3 (or the LLC). The L1 and L2 caches are usually private to each core and the LLC is shared among all cores of processor. The LLC is much larger (megabytes size) than the L1 and L2 caches (kilobytes size). Cache memories are divided into equal blocks called ways and consisting of cache lines. Cache lines with the same index in ways form a set. When the processor needs either to read or write data to a specific location in RAM, it first checks whether a copy of data is already present in one of the cache levels. If the data is found, a cache hit is triggered and the CPU performs operations on the cache quickly in a few clock cycles. Otherwise a cache miss occurred and the processor inevitably brings data from RAM at a much slower pace. There are two cache architectures in modern processors: inclusive and exclusive. Exclusive caches do not store redundant copies, hence enable higher cache capacity. For instance, exclusiveness is widely used in AMD Athlon microarchitectures. Otherwise, in inclusive architectures, all cache lines in higher levels are also available in the lower level. For instance, the architecture of LLC in Intel's Nehalem processors is inclusive to reduce snoop traffic between cores and the processor sockets [8]. Inclusiveness provides less cache capacity but offers higher performance to the processor.

\section{B. Cache-based Side-channel Attacks}

The virtualization layer enables running multiple VMs on a physical machine. Main physical resources of the host such as CPU and RAM are shared between VMs through the virtualization layer, which has a strong security impact on running VMs. Furthermore, such sharing is strongly at odds with isolation, both at the hardware level and at the software level. To make better use of shared resources, some optimization techniques such as memory page deduplication are used in the virtualization layer. These techniques may have an impact on isolation directly or indirectly. In such environment, processor's caches and especially the LLC are shared among running VMs or virtualized instances. These caches are widely exploited through cache-based side-channel attacks. Because the LLC is shared among all cores of processor, it is more exploited in the case of side-channel attacks between two VMs. The main idea of side-channel attacks rests on the eviction of cache lines from cache and measuring fetching time of memory lines from RAM to know which cache lines are re-accessed by the victim during an operation, to obtain the victim's cache access pattern. Furthermore, if a memory line was flushed by 
the victim from the cache, it takes more $\mathrm{CPU}$ cycles to fetch the line from the main memory when it is reaccessed. Otherwise, the line is already in the cache, thus requiring fewer CPU cycles. This timing difference is exploited to profile cache access patterns of the victim. Generally, any cache-based side channel attack consists of three principal steps: (1) the attacker evicts cache lines of victim VM from processor's cache by e.g., filling cache sets (2) the attacker then waits for a given period of time (several CPU cycles) while the victim is doing some operations (3) finally, the attacker re-accesses the same cache lines which are evicted from caches, to know which ones are used by the victim during the execution of the victim's operations e.g., encryption.

There are three classes of the attacks: time-driven, trace-driven, and access-driven. The main idea behind time-driven attacks is to measure the execution time of e.g., an encryption operation, and derive a secret key from the gathered samples by finding any correlation between measured time and the operation. In tracedriven attacks [10], [11], the attacker inspects cache activities (i.e., memory lines which are accessed) of the victim during its execution in order to obtain a sequence of cache hits and cache misses. For instance, monitoring which memory accesses to a lookup table lead to cache hits, allows finding indices of tables entries. In accessdriven attacks [3], [9], the attacker tries to find any relation between $e . g$., an encryption process and accessed cache lines, to exploit the pattern of cache accesses of the victim. Technically, we may also distinguish different techniques to perform a cache-based side-channel attack: Prime + Probe, Flush + Reload, Flush + Flush, and Evict+Time. Prime +Probe is the first and common technique for profiling the victim VM through a shared cache. The attacker needs to observe which cache lines are accessed by the victim during the execution of sen- sitive operations using timing information (provided by instructions such as rdtsc). The Flush+Reload technique has more resolution than other attack techniques because of leveraging memory deduplication feature and inclusive caches.

\section{DESIGN AND IMPLEMENTATION}

The main idea behind our detection approach is to analyze periodically the behavior of running VMs, to identify malicious activities which may be detected as a side-channel attack. We need to have several different fine-grained (provided by the hardware) information gathered along the execution of VMs. To date, there are two hardware sources providing high resolution cache-related information on running VMs that might be utilized for analysis purposes: Intel CMT and HPCs.

\section{A. Threat Model}

We assume a IaaS cloud and that the underlying CPU is based on $\mathrm{x} 86$ architecture equipped with Intel CMT. We also assume that victim and attacker VMs are pinned to different cores of processor. It means only the LLC is shared between two VMs. Thus private resources of a core such as L1 cache and the unified L2 cache are not shared between two VMs. The detection module aims to detect side-channel attacks conducted between two VMs through the LLC. We further assume that the attacker has full control on his VM and is capable of exploiting the LLC through certain lowlevel instructions such as rdtsc and clflush which are accessible to unprivileged users in $\mathrm{x} 86$ architecture. Design and implementation objectives for our detection are as follows: (1) it should detect side-channel attacks between two VMs through the LLC (2) it should not impose modifications to the hypervisor to be generic and compatible with other hypervisors (3) it should induce small performance overhead. 


\section{B. Components of the Detection Module}

In our design, we take advantage of following techniques/technologies to detect the attacks:

\section{a) Intel Cache Monitoring Technology (CMT):}

Intel has introduced two features in processors to improve cache utilization: Cache Allocation Technology (CAT) and Cache Monitoring Technology (CMT). CAT is basically intended to provide Quality of Service (QoS) to running workloads (i.e., VMs, applications) in the allocation of LLC. CMT aims for monitoring the use of shared resources. Recent processors have several cores allowing to run simultaneously diverse workloads on CPU cores. This capability may potentially cause run-time resource contention on shared processor resources such as the LLC. Consequently, such resource contention may result in reduced performance of the overall system. This issue gets more importance in real-time and virtualized systems, where performance is critical. For instance, in a virtualized environment such as the cloud, CMT allows monitoring LLC occupancy of VMs running on the same physical host in a data center, finding aggressive VMs and then reacting to such events by applying resource usage limitation through CAT. Regarding cache-based side-channel attacks, as the attacker VM evicts several cache lines many times

\begin{tabular}{|c|c|}
\hline Name & Event Description \\
\hline LLC-misses & Last-level cache misses \\
\hline LLC-references & Last-level cache references \\
\hline LLC-r-accesses & Last-level cache read accesses \\
\hline LLC-w-accesses & Last-level cache write accesses \\
\hline L1-dcache-misses & L1-data cache misses \\
\hline L1-icache-misses & L1-instruction cache misses \\
\hline iTLB-r-accesses & Instruction TLB read accesses \\
\hline iTLB-cache-misses & Instruction TLB read misses \\
\hline
\end{tabular}

Table I: Specific hardware performance events related to cache from the cache, this aggressive behavior can be detected by CMT. Hence cache occupancy is provided at the byte-level granularity. Therefore, we can use the cache occupancy of a VM as an effective information in the detection of cache-based side-channel attacks through the LLC. In our detection module, we thus leverage CMT as a means to provide useful information to detect these attacks targeting the LLC. To date, our detection approach is the first to leverage Intel CMT to detect side-channel attacks.

\section{b) Hardware Performance Counters (HPCs):}

These are special platform hardware registers used to store statistics about various CPU events such as clock cycles and context-switches. Those events are widely used to profile a program's behavior to analyze and optimize it. Collected performance data may also be analyzed to detect any situation of performance degradation. For instance, in the Intel micro-architecture, the events are provided by Processor Monitoring Units $(P M U)$ and are accessible through the perf_events subsystem provided by the Linux kernel. For system security, HPCs are widely used in the detection of different attacks such as Rowhammer [12] and cachebased side-channels [4]. Our detection module takes advantage of HPCs to detect malicious VMs in a virtualized environment. Table I] gives an overview of several hardware cache events that might be utilized in the detection of cache-based side-channel attacks in the system. For real-time attack detection, the detection module should simultaneously get access to events. Therefore, we select the most effective events among those available for the attack detection. The detection module aims to detect any malicious behavior induced by a virtual machine, in the cache. We thus focus on the events which are necessarily related to the LLC. Previous 
works [4] [6] [13] proposed to focus on LLC-misses and LLC-references as the important events to monitor. After our observation during experimentation, in our approach, we use LLC-misses, LLC-references, iTLB-cache-misses, and iTLB-r-accesses as the most effective performance counters to detect the attack.

c) Anomaly Detection: As an application class of Machine Learning, the Gaussian anomaly detection approach detects data points in a data set which do not fit well with the rest of the data set. This approach is widely used in monitoring and diagnostic applications. Given a data set $X$ with $m$ data points. Each data point $x^{(i)}$ in the $X$ is represented by $n$ features. Among data points in the data set, there might be some anomalous data points to detect. The first step in the Gaussian method is to fit a Gaussian distribution to data points of data set. We thus calculate the mean $\left(\mu_{j}\right)$ and the variance $\left(\sigma^{2}{ }_{j}\right)$ of each feature $j=1, \ldots, n$ for $x_{j}^{(1)}, \ldots, x_{j}^{(m)}$, to estimate Gaussian distribution of features.

Finally, according to a defined threshold value based on a cross-validation, we can find anomalous data points through calculating the probability of each data point $\left(p\left(x^{(i)}\right)\right)$ and finding those with lower probability value than threshold. To find an optimal threshold, we use a cross-validation set and calculate the $F_{1}$-score [14] for each example in the set. We calculate the $F_{1}$-score using the precision (prec) and recall (rec), where $t p$ is the number of true positives, $f p$ is the number of false positives, and $f n$ is the number of false negatives.

$$
\begin{gathered}
p\left(x ; \mu, \sigma^{2}\right)=\frac{1}{\sqrt{2 \pi \sigma^{2}}} e^{-\frac{(x-\mu)^{2}}{2 \sigma^{2}}} \\
F_{1}=2 \cdot \frac{\text { prec.rec }}{\text { prec }+ \text { rec }} \quad \text { prec }=\frac{t p}{t p+f p} \quad \text { rec }=\frac{t p}{t p+f n}
\end{gathered}
$$

In our detection approach, each virtual machine is represented as a data point in the data set, and performance information such as LLC-misses and llc-occupancy are the features of each data point. We apply Gaussian anomaly detection on the data set to detect any anomalous VM. As there is a limited number of running VMs on a physical machine, the data set size remains small and will efficiently reduce the computation cost of the anomaly detection module.

\section{Implementation}

Fig. 1 gives an overview of the architecture of the detection module. Our detection prototype is composed of three threads: The main thread is the core of module and probes periodically the system to collect statistics information (i.e., LLC-occupancy, LLC-misses, etc.) of all running VMs. A second thread scans the host machine to provide a live list of running VMs. A detection thread applies Gaussian anomaly detection on collected information to identify any malicious behavior. The detection module is implemented as a user space program written



Figure 1: Detection module architecture 
in $\mathrm{C}$, and operates on the KVM/QEMU hypervisor.

\section{Evaluation}

To validate our detection approach, we ran $6 \mathrm{VMs}$ on the following experimental platform: Intel(R) Xeon CPU D-1541 2.10GHz, 16GB RAM, L1 $32 \mathrm{~KB}$, L2 $256 \mathrm{~KB}$, LLC $12 \mathrm{MB}$.

\section{A. Evaluation Process}

1) Attack Scenario: We use the code of Prime+Probe attack provided by [13] [15]. We consider one attacker VM and one victim VM. We assume that other VMs perform normal operations and among them there is a VM with a compute-intensive workload. We need a compute-intensive workload (CIW) to determine if the detection module is able to distinguish between a malicious VM and a honest compute-intensive VM (CIW-VM).

2) Experimental Scenarios: In our evaluation, we experiment different scenarios for the virtualized platform.

a) No-attack, No-CIW: In this scenario, two VMs are in idle status i.e., the attacker does not perform any side-channel attack and there is not any computeintensive workload. As shown in Fig. 5]a, almost all VMs have roughly the same values for the perf events. We can also see that the attacker VM, victim VM, and other VMs have normal values for llc-occupancy (comparing to the attack situation). All VMs have the same LLC-misses and $i \mathrm{TLB}-\mathrm{cache}-\mathrm{misses}$ rates.

b) Attack, No-CIW: In this scenario, the attacker performs the side-channel attack and there is not any running compute-intensive workload. As we can see on Fig. 5.b, the attacker has a lot of LLC-misses and occupies more LLC space than other VMs. The attacker also encounters fewer iTLB-cache-misses than other VMs. Indeed, as seen before, the attacker VM re-accesses the same memory lines several thousands times during the attack period. Consequently, read access to TLB cache results in cache hit.

c) No-attack, CIW: In this scenario, the attacker VM does not perform any attack. Among other VMs, one VM performs compute-intensive operations. As shown in Fig. 5.c, the compute-intensive VM has very different perf and llc-occupancy values. This VM has a higher value for $11 \mathrm{c}$-occupancy than other VMs and a large number of LLC-misses. This behavior is not abnormal for a compute-intensive workload. However, it has larger iTLB-cache-misses (also iTLB-cachemisses ratio) than the attacker VM in the attack scenario. In fact, the compute-intensive VM has a larger instruction footprint that results in increasing instruction cache misses. This difference may be considered as an important feature to distinguish between attacker and compute-intensive VMs.

d) Attack, CIW: In this scenario, the attacker VM performs a side-channel attack. Another VM performs compute-intensive operations as well. As we can see on Fig. 5 5 , the attacker and compute-intensive VMs have a larger number of LLC-misses and llc-occupancy than other VMs. Although, two VMs have a large value of LLC-misses, the attacker VM has a higher distinct value than the compute-intensive VM. On the other hand, the compute-intensive VM has a larger value for $11 \mathrm{c}-$ occupancy than the attacker VM. We also observe that the attacker VM encounters fewer iTLB-cache-misses than the computeintensive VM. This significant difference may be used to distinguish the attacker VM from the compute-intensive VM. This scenario may thus potentially generate false positives alerts. 


(a) no attack, no CIW
(b) attack, no CIW
(c) no attack, CIW
(d) attack, CIW



Figure 2: Perf information of VMs in different scenarios

3) Origin of High Perf Values: To justify that the high number of e.g., cache-misses measured for attacker VM, are generated by the spy program running in the attacker VM, we use perf-kvm. This is another extension of perf that allows tracing/measuring a guest OS running on top of KVM. As shown in Table. III. clear_page_c_e is the first kernel function that generates a lot of LLC-misses during the execution of spy program. After verification on the guest OS, we observe that this function is called many times during the spy program execution. This shows that a process (in the attacker VM) is spending its time to clear memory. Such a situation particularly occurs during a cachebased side-channel attack, especially in the first phase of the attack i.e., evicting memory lines from cache. We also verified the rate of LLC-misses when the spy program was not executing and it was $\simeq 1.5 \%$ (see Fig. 2). We can thus confirm that the high rate of LLC-misses is induced by the spy program.



Figure 3: LLC cache misses vs. system functions for attacker VM

Additionally, as shown in Fig. 3, during a sidechannel attack, the rate of $11 \mathrm{c}$-occupancy increases abnormally because of evicting cache lines from LLC by the spy program, early in the first phase $(t=3 s)$ of the attack. This event is more notable in the case of the attacks based on Prime+Probe or Evict+Reload techniques when the attacker uses an eviction set to evict totally a cache set. 


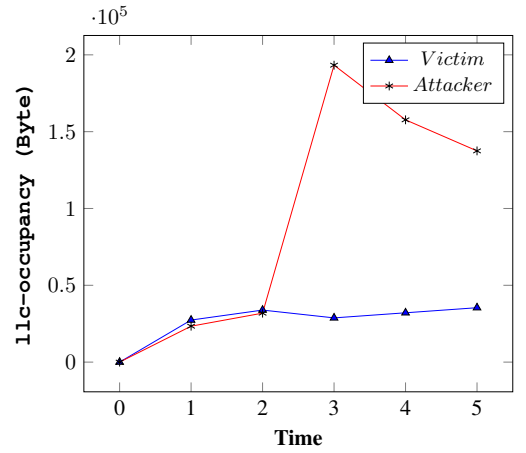

Figure 4: LLC occupancy of attacker and victim VMs during the attack

\begin{tabular}{|c|c|c|}
\hline Overhead & shared Object & Symbol \\
\hline $15.92 \%$ & [guest.kernel] & [g] clear_page_c_e \\
\hline $6.68 \%$ & [guest.kernel] & [g] page_add_new_anon_rmap \\
\hline $1.88 \%$ & [guest.kernel] & {$[\mathrm{g}]$ _schedule } \\
\hline $1.62 \%$ & [guest.kernel] & {$[\mathrm{g}]$ _switch_to } \\
\hline $1.44 \%$ & [guest.kernel] & {$[\mathrm{g}]$ tcp_back } \\
\hline $1.38 \%$ & [virtio_ring] & [g] virtqueue_get_buf \\
\hline $1.32 \%$ & [virtio_net] & [g] virtnet_poll \\
\hline
\end{tabular}

Table II: Output of perf-kvm for the attacker VM: top functions generating cache misses in the attacker VM

\section{B. Evaluation Results}

In this Section, we present detection accuracy and performance overhead results.

1) Detection Accuracy: To evaluate the accuracy of cache-based side-channel attack detection, we measure the true positive rate and false positive rate generated



Figure 5: Roc curve without \& with Intel CMT by our detection module in the different scenarios described in Section IV] Results show that the detection module works very well in experimental scenarios (a) and (b), and detects malicious VM correctly. However, our detection module may generate false positives in scenario (c) and especially in scenario (d) i.e., when a compute-intensive workload is present. In Fig. 5 c and Fig. 5d, we also notice that there is a clear difference between the iTLB-cache-misses rate $\left(\frac{\text { iTLB-cache-misses }}{\text { ITLB-r-accesses }}\right)$ of the compute-intensive VM and the attacker VM. Therefore, this can be used as an important feature for distinguishing between the attacker VM and compute-intensive VM. We also observe that a malicious VM has a lower iTLB-cache-misses rate (e.g., 1.2\%) than a benign VM (e.g., 30\%). Consequently, by using this feature, we can distinguish compute-intensive workload and malicious VMs, that results in a false positive reduction. We also carried out other experimentation to assess the efficiency of Intel CMT in detection accuracy on two different scenarios; when Intel CMT is enabled, and when it is not. We obtained 0.67 as $F_{1}$ - score when Intel CMT was enabled than 0.5 without Intel CMT. It means, we effectively obtained more precision in our detection approach through leveraging Intel CMT (Fig. 4).

2) Performance Overhead: The detection module imposes about $2 \%$ performance overhead to the hypervisor, which is negligible. However, we measured this value for 6 running VMs on the platform. This value may increase when extending the number of running VMs or during a long period of probing to collect perf information.

\section{CONCLUSION}

In this paper, we presented a new approach of crossVM side-channel attack detection leveraging Intel Cache 

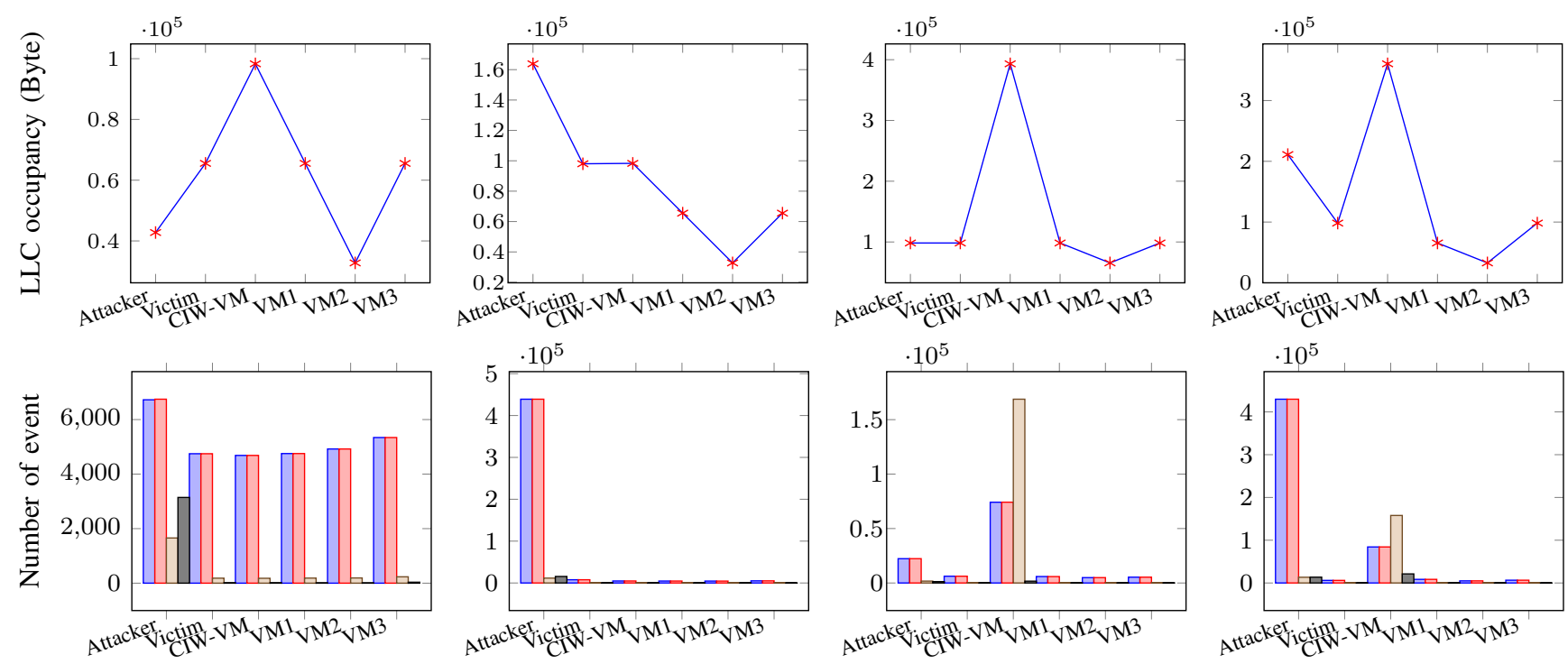

(a) no attack, no CIW

(b) attack, no CIW

(c) no attack, CIW

(d) attack, CIW

Figure 6: Perf information of VMs in different scenarios

Monitoring Technology (CMT), Hardware Performance

Counters (HPCs), and the Gaussian anomaly detection method to detect malicious VMs in IaaS platforms. This work has demonstrated the promising performance of Intel CMT and HPCs in the detection of side-channel attacks in a virtualized environment. As future work, we will explore improving our approach, also comparing it with emerging approaches for side-channel attacks detection.

\section{REFERENCES}

[1] T. Ristenpart, E. Tromer, H. Shacham, and S. Savage, "Hey, you, get off of my cloud: exploring information leakage in third-party compute clouds," in Proceedings of the 16th ACM conference on Computer and communications security, pp. 199-212, ACM, 2009.

[2] G. Irazoqui, T. Eisenbarth, and B. Sunar, "S\$a: A shared cache attack that works across cores and defies vm sandboxing-and its application to aes," in 2015 IEEE Symposium on Security and Privacy, pp. 591-604, IEEE, 2015.

[3] Y. Yarom and K. Falkner, "Flush+Reload: A High Resolution, Low Noise, L3 Cache Side-Channel Attack," in 23rd USENIX Security Symposium, pp. 719-732, 2014.
[4] M. Chiappetta, E. Savas, and C. Yilmaz, "Real time detection of cache-based side-channel attacks using hardware performance counters," Applied Soft Computing, vol. 49, pp. 1162-1174, 2016.

[5] T. Zhang, Y. Zhang, and R. B. Lee, "Cloudradar: A real-time side-channel attack detection system in clouds," in International Symposium on Research in Attacks, Intrusions, and Defenses, pp. 118-140, Springer, 2016.

[6] M. Payer, "Hexpads: a platform to detect stealth attacks," in International Symposium on Engineering Secure Software and Systems, pp. 138-154, Springer, 2016.

[7] R. O. Duda, P. E. Hart, and D. G. Stork, Pattern classification. John Wiley \& Sons, 2012.

[8] M. E. Thomadakis, "The architecture of the nehalem processor and nehalem-ep smp platforms," Resource, vol. 3, p. 2, 2011.

[9] D. Gruss, C. Maurice, K. Wagner, and S. Mangard, "Flush+ Flush: A Fast and Stealthy Cache Attack," arXiv preprint 1511.04594, 2015.

[10] O. Acriçmez and Ç. K. Koç, "Trace-driven cache attacks on aes (short paper)," in International Conference on Information and Communications Security, pp. 112-121, Springer, 2006.

[11] J.-F. Gallais, I. Kizhvatov, and M. Tunstall, "Improved tracedriven cache-collision attacks against embedded aes implementations," in International Workshop on Information Security Applications, pp. 243-257, Springer, 2010.

[12] M. Seaborn and T. Dullien, "Exploiting the dram rowhammer 
bug to gain kernel privileges," Black Hat, 2015.

[13] D. Gruss, C. Maurice, K. Wagner, and S. Mangard, "Flush+ flush: a fast and stealthy cache attack," in Detection of Intrusions and Malware, and Vulnerability Assessment, pp. 279-299, Springer, 2016.

[14] D. M. Powers, "Evaluation: from precision, recall and f-measure to roc, informedness, markedness and correlation," 2011.

[15] "Flush+Flush side-channel attack github repository, https:// github.com/iaik/flush_flush," 